\title{
The influence of plasmon resonance on the decrease of resonatorless laser generation thresholds
}

\author{
Valeriy A. Donchenko ${ }^{\mathrm{a}, \mathrm{b}}$, Aleksey A. Zemlyanov ${ }^{\mathrm{a}, \mathrm{b}}$, Michael M. Zinovjev ${ }^{\mathrm{b}}$, Anna N. Panamaryova ${ }^{\mathrm{c}}$, \\ Vladimir A. Kharenkov ${ }^{\mathrm{a}, \mathrm{b}}$

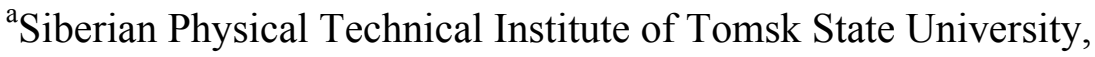 \\ bational Research Tomsk State University, \\ ${ }^{\mathrm{c}}$ National Research Tomsk Polytechnic University \\ don@spti.tsu.ru,zem116@mail.ru,muxa9229@gmail.com, \\ n.panamarev@mail.ru, harvoll@mail.ru
}

\begin{abstract}
The paper presents the results of the measurements of the values of resonatorless laser generation power threshold densities in thin layers of $6 \mathrm{G}$ rhodamine colloidal solutions with single plasmon-resonance gold and silver nanoparticles and non-plazmon resonance nanoparticles of platinum and zinc oxide. It is shown that when gold, platinum and silver nanoparticles are used, the thresholds values are approximately equal at the same concentrations.
\end{abstract}

Keywords: plasmon resonance, resonatorless generation, nanoparticles, laser dye, active medium.

Currently the direction associated with researches of resonatorless laser generation in active media with nanoparticles is actively developing. In international scientific literature this direction is known as random lasing (random, stochastic, chaotic generation) [1]. Within this direction the questions related to the laser generation in the active nano-dispersed media such as, for example, solutions and polymer films with laser dyes and embedded nanoparticles of various materials are studied. Also, composite laser-active media present great interest because lasing thresholds in such active media are significantly lower than in the same active media without nanoparticles. The physical reason of laser generation in scattering media with laser-active molecules is considered to be the formation of positive feedback due to multiple scattering of stimulated secondary radiation in a scattering excited active medium [2]. Another reason is considered to be a local increase in the power density of the optical field generated in the near field of a nanoparticle or a cluster of nanoparticles when plasmon resonance is realized, i.e. the coincidence of the incident or the secondary radiation with intrinsic frequencies of the nanoparticle or the cluster of nanoparticles [3,4]. In this case, we can also expect an increase in the speed of transitions in emitting molecules [5] what may lead to a decrease in lasing generation threshold and increase in its efficiency.

Nowadays in scientific literature there are no works devoted to the comparison of the resonatorless generation characteristics of the dyes solution with nanoparticles having plasmon resonance properties at the wavelength

22nd International Symposium Atmospheric and Ocean Optics: Atmospheric Physics, edited by

Gennadii G. Matvienko, Oleg A. Romanovskii, Proc. of SPIE Vol. 10035, 1003526 .

(C) 2016 SPIE - CCC code: $0277-786 X / 16 / \$ 18 \cdot$ doi: $10.1117 / 12.2249339$

Proc. of SPIE Vol. 10035 1003526-1 
of the exciting radiation (for example, gold) and of the dyes solution with nanoparticles which do not have plasmon resonances under the same conditions.

The aim of this work is an experimental study of the effect of plasmon-resonance gold and silver nanoparticles and non-plazmon-resonance platinum and zinc oxide nanoparticles on the value of the resonatorless generation thresholds in colloidal ethanol solutions of laser-active R6G dye.

In the experiment to excite the active medium the second harmonic radiation (wavelength $\lambda=532 \mathrm{~nm}$, pulse duration is $6 \mathrm{~ns}$ ) YAG: $\mathrm{Nd}^{3+}$ of the laser LOTIS-TII was used.

Colloid solutions of gold, silver, platinum and zinc oxide were obtained by laser ablation [6] in ethyl alcohol. Dimensions of the used nanoparticles were determined by electron microscopy. The mean value of the diameter of both materials nanoparticles is $10 \mathrm{~nm}$. Figure 1 shows the calculations results of the absorption and scattering cross sections spectral distributions of the used nanoparticles in ethanol made in accordance with Mie theory.
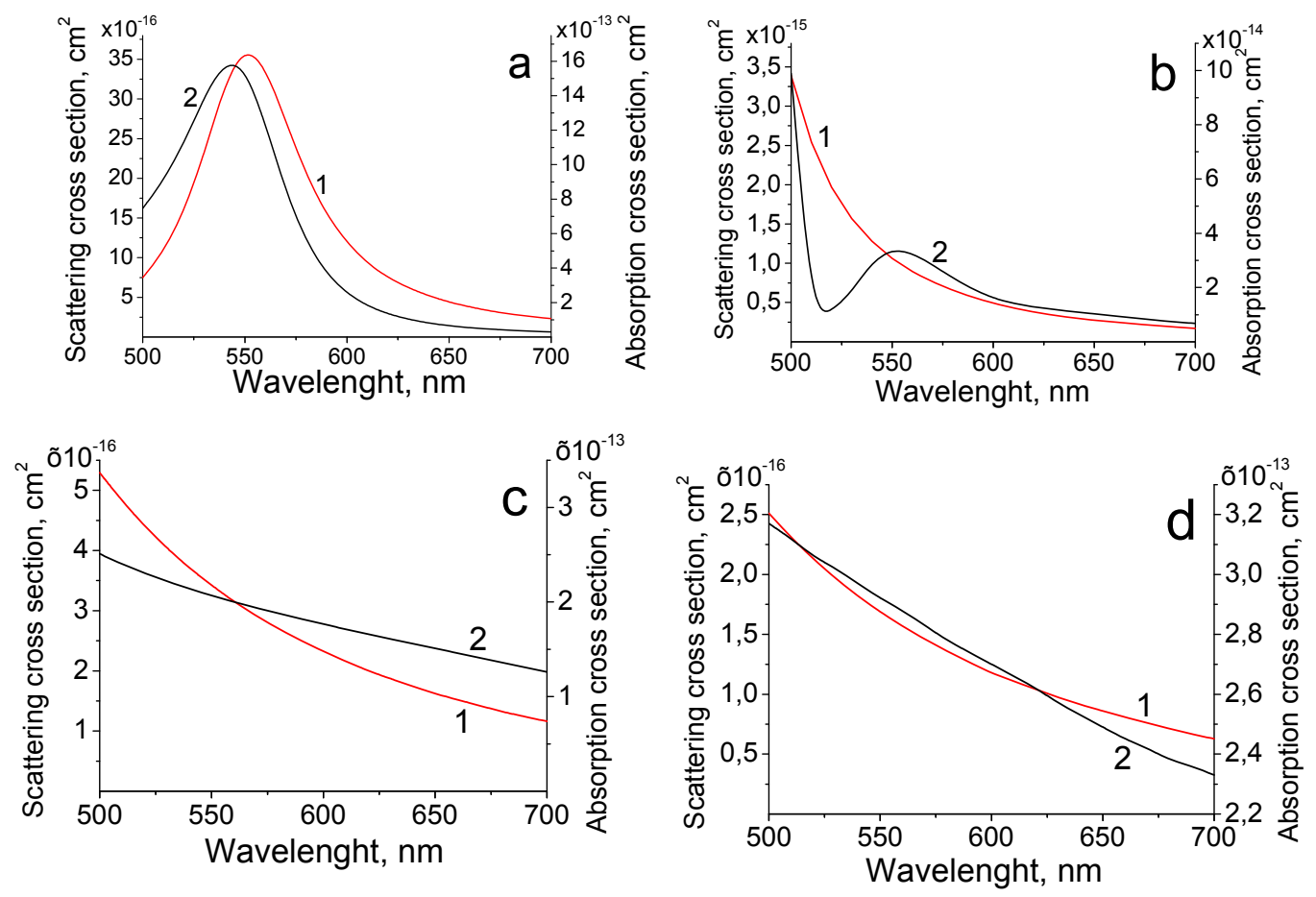

Figure 1. The calculated spectra of the cross-sections of the nanoparticles scattering (1) and absorptions (2): $a-A u ; b$ -

$$
\mathrm{Ag} \text {; c - Pt; d - ZnO }
$$

It is seen that only gold nanoparticles have a strongly marked plasmon resonance at the wavelength of $532 \mathrm{~nm}$, the absorption spectrum of silver nanoparticles only slightly overlaps with the excitation wavelength (532 $\mathrm{nm})$ and has a certain feature at R6G luminescence wavelength $(560 \mathrm{~nm})$. Platinum and zinc oxide nanoparticles do not have spectral features at the wavelengths of $532 \mathrm{~nm}$ and $560 \mathrm{~nm}$ (maximum of the R6G solution luminescence spectrum).

Figure 2,a shows the attenuation spectra of colloidal solutions obtained on a Shimadzu UV-3600 spectrophotometer. 

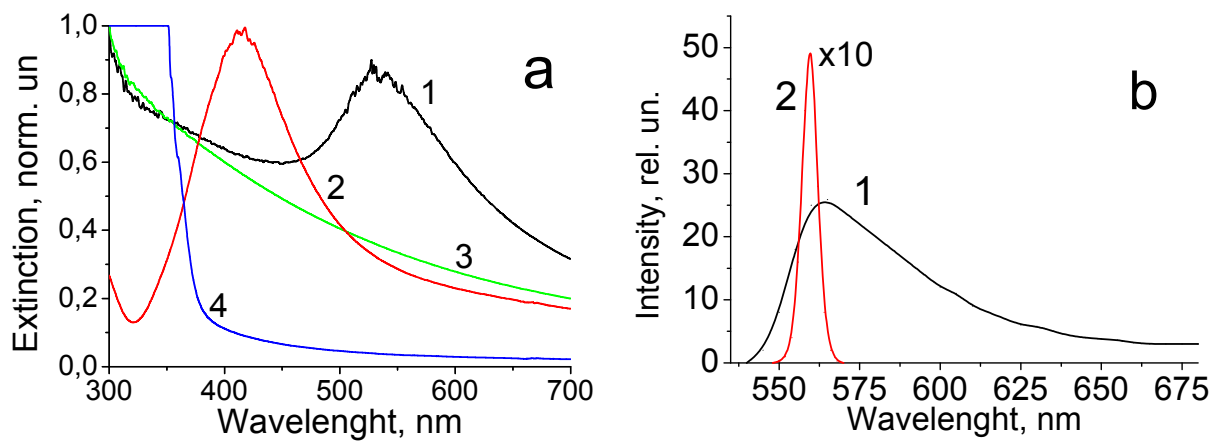

Figure 2. The normalized attenuation spectra (a) of colloidal solutions with nanoparticles $\mathrm{Au}$ (1), $\mathrm{Ag}(2), \mathrm{Pt}(3)$, $\mathrm{ZnO}(4) ; \mathrm{b})$ the spontaneous luminescence (1) and generation (2) spectra

Figure 1 and Figure 2,a show that the calculated absorption and scattering spectra of the nanoparticles are qualitatively similar to the measured attenuation spectra of the nanoparticles used in the experiment. No features are observed at the wavelength of $560 \mathrm{~nm}$ in the silver attenuation spectrum.

In the experiment, the R6G solution with $10-3 \mathrm{~mol} / \mathrm{L}$ concentration and nanoparticles was placed between glass slides with an active layer thickness of about 20 microns. The laser excitation beam diameter in the active medium was $2 \mathrm{~mm}$. Continuous adjustment of the excitation power was carried out with a Glan-Thompson prism. The radiation receiver was an AVASPEC ULS2048L-USB2 spectrometer.

As results of the experiments, the spectral-energy characteristics of the generation in thin layers (20 microns) of R6G solutions doped with nanoparticles have been obtained. Figure 2,b shows the typical spectra of spontaneous luminescence and generation. The excitation power density value corresponding reduction of the solution emission spectrum by 2 times was used as the generation threshold value. As a result of the measurements of the generation threshold values the dependence of the threshold excitation power density on the volume fraction of nanoparticles (Figure 3) has been obtained.

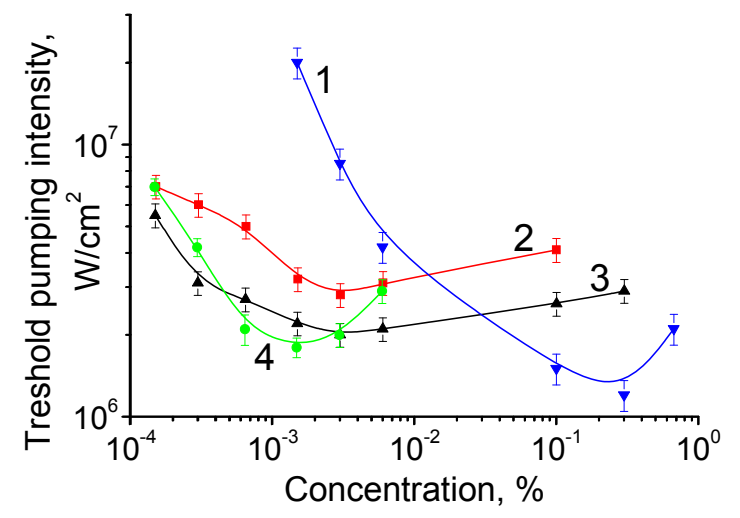

Figure 3. The dependence of the threshold excitation power density on the volume fraction of the nanoparticles in the active solution: 1 - $\mathrm{ZnO} ; 2$ - $\mathrm{Pt} ; 3$ - $\mathrm{Au} ; 4$ - $\mathrm{Ag}$ 
Superluminescence of the layer 20 micrometers thick of R6G dye solution without nanoparticles is observed when the excitation power densities are $\sim 10^{8} \mathrm{~W} / \mathrm{cm}^{2}$. It is important that in the case of adding the nanoparticles to the active medium the generation threshold is reduced by two orders of magnitude. The results of the experimental comparison of lasing generation thresholds values in the thin layer of the R6G dye when non-plasmon resonance platinum, zinc oxide nanoparticles and plasmon-resonance nanoparticles of gold and silver are added and the excitation is at $532 \mathrm{~nm}$ wavelength shows that for the used nanoparticles the role of plasmon resonance in the effect of thresholds decrease of laser generation without resonance is not significant.

Thus, despite the realization of plasmon resonance at the excitation wavelength $\sim 530 \mathrm{~nm}$ in a colloidal R6G solution with $\mathrm{Au}$ nanoparticles, the generation thresholds are only 1.5-2 times lower than when Pt nanoparticles not having plasmon-resonance properties in the visible spectrum are added. While theoretical calculations [5] predict an increase in the local field intensity by orders of magnitude with respect to intensity of the incident field in the case of plasmon resonance.

\section{REFERENCES}

[1] Lawandy, N. M., Balachandran, R. M, “ Random laser?” Nature. Vol. 373, № 6511, 204-208 (1995).

[2] Cao, H. "Lasing in random laser" in [Waves Random Media. Topical Review], Vol 13. R1-R39 (2003).

[3] Khlebtsov, N.G. "Optics and biophotonics of nanoparticles with plasmon resonance", Quantum Electronics. Vol. 38, № 6, 504-529 (2008).

[4] Noginov M.A., Zhu G., Bahaura M., Adegoke J., Small C., Ritzo B.A., Drachev V.P., Shalaev V.M. “The effect of gain and absorption on surface plasmon in metal nanoparticles”, Applied Physics. B. Vol. 86, № 3, 455-460, (2007).

[5] Klimov, V.V., Guzatov, D.V. "Optical properties of an atom in the presence of a cluster of two nanospheres", Quantum Electronics, Vol. 37, № 3, 209-230 (2007).

[6] Svetlichnyi V.A., Lapin I.N. "Structure and properties of nanoparticles fabricated by laser ablation of Zn metal targets in water and ethanol”, Russian Physics Journal, Vol. 56. № 5, 581-587 (2013).

This work was supported by Ministry of Education and Science of the Russian Federation (task №2014/223, project code: 1347). 